\title{
Two unusual cases of successful treatment of hypermucoviscous Klebsiella pneumoniae invasive syndrome
}

Hiroki Namikawa ${ }^{1,2}$, Koichi Yamada ${ }^{1}$, Hiroki Fujimoto ${ }^{1}$, Ken-Ichi Oinuma ${ }^{3}$, Yoshihiro Tochino², Yasuhiko Takemoto ${ }^{2}$, Yukihiro Kaneko ${ }^{3}$, Taichi Shuto ${ }^{2}$ and Hiroshi Kakeya ${ }^{1 *}$ (1)

\begin{abstract}
Background: A few Japanese cases of hypermucoviscous Klebsiella pneumoniae (K. pneumoniae) invasive syndrome have recently been reported. Although extrahepatic complications from bacteremic dissemination have been observed, infected aneurysms are rare. Furthermore, the primary source of infection is generally a liver abscess, and is rarely the prostate. Therefore, we report two atypical cases of hypermucoviscous K. pneumoniae invasive syndrome.

Case presentation: The first case was an 81-year-old Japanese man with no significant medical history, who was referred to our hospital for vision loss in his right eye. Contrast-enhanced whole-body computed tomography revealed abscesses in the liver and the prostate, and an infected left internal iliac artery aneurysm. Contrastenhanced head magnetic resonance imaging revealed brain abscesses. Cultures of the liver abscess specimen and aqueous humor revealed K. pneumoniae with the hypermucoviscosity phenotype, which carried the magA gene (mucoviscosity-associated gene A) and the rmpA gene (regulator of mucoid phenotype A). We performed enucleation of the right eyeball, percutaneous transhepatic drainage, coil embolization of the aneurysm, and administered a 6-week course of antibiotic treatment. The second case was a 69-year-old Japanese man with diabetes mellitus, who was referred to our hospital with fever, pollakiuria, and pain on urination. Contrast-enhanced whole-body computed tomography revealed lung and prostate abscesses, but no liver abscesses. Contrastenhanced head magnetic resonance imaging revealed brain abscesses. The sputum, urine, prostate abscess specimen, and aqueous humor cultures revealed K. pneumoniae with the hypermucoviscosity phenotype, which carried magA and rmpA. We performed enucleation of the left eyeball, percutaneous drainage of the prostate abscess, and administered a 5-week course of antibiotic treatment.
\end{abstract}

Conclusions: Hypermucoviscous K. pneumoniae can cause infected aneurysms, and the prostate can be the primary site of infection. We suggest that a diagnosis of hvKP invasive syndrome should be considered in all patients who present with $K$. pneumoniae infection and multiple organ abscesses.

Keywords: Hypermucoviscous Klebsiella pneumoniae, Abscess, Infected aneurysm, Endophthalmitis, Prostate, magA, rmpA, String test

\footnotetext{
* Correspondence: kakeya@med.osaka-cu.ac.jp

${ }^{1}$ Department of Infection Control Science, Osaka City University, Graduate

School of Medicine, 1-4-3, Asahi-machi, Abeno-ku, Osaka 545-8585, Japan

Full list of author information is available at the end of the article
}

(c) The Author(s). 2016 Open Access This article is distributed under the terms of the Creative Commons Attribution 4.0 International License (http://creativecommons.org/licenses/by/4.0/), which permits unrestricted use, distribution, and reproduction in any medium, provided you give appropriate credit to the original author(s) and the source, provide a link to the Creative Commons license, and indicate if changes were made. The Creative Commons Public Domain Dedication waiver (http://creativecommons.org/publicdomain/zero/1.0/) applies to the data made available in this article, unless otherwise stated. 


\section{Background}

Klebsiella pneumoniae (K. pneumoniae) is a common pathogen in community-acquired and nosocomial infections [1]. However, a new type of $K$. pneumoniae invasive syndrome has been identified in southeast Asia during the last 2 decades [2]. In the 1980s, a community-dwelling Taiwanese patient presented with a primary liver abscess that involved $K$. pneumoniae [3]. In Japan, a few recent studies have reported pyogenic infections that were caused by $K$. pneumoniae $[4,5]$. Extrahepatic complications have also been observed because of bacteremic dissemination, such as endophthalmitis, meningitis, and other diseases. However, infected aneurysms caused by hypermucoviscous $K$. pneumoniae (hvKP) are relatively rare. A liver abscess may occur after leakage of K. pneumoniae from the intestinal epithelium and bacterial translocation into the liver via the portal circulation [6]. However, hvKP bacteremia involving the prostate via the ascending route is extremely rare. In this context, hypermucoviscous strains are identified using the appearance of colonies that are grown on an agar plate. Furthermore, several studies have indicated that hypermucoviscosity is associated with the mucoviscosity-associated gene $\mathrm{A}(m a g A)$ and the regulator of mucoid phenotype $\mathrm{A}(\operatorname{rmp} A)$ genes $[7,8]$.

We report two rare cases of hvKP invasive syndrome that involved strains that were positive for $m a g A$ and $r m p A$. The first case involved an 81-year-old man with hvKP invasive syndrome who presented with brain, liver, and prostate abscesses; bacterial endophthalmitis; and an infected aneurysm. The second case involved a 69-yearold man with hvKP invasive syndrome who presented with brain, lung, and prostate abscesses; bacterial endophthalmitis; but no liver abscesses.

\section{Case presentation}

\section{Case 1}

An 81-year-old man was referred to our hospital with a 10-day history of vision loss in his right eye. He also exhibited ophthalmalgia and general malaise. There was no relevant medical history, no history of alcoholism, and no use of prescription medication. He had no recent travel history, including within Southeast Asia, and no history of contact with animals. A physical examination revealed that his vital signs were stable, although he had swelling of the right eyelid and corneal edema in the right eye (Fig. 1a). Blood tests revealed a white blood cell count of $11,700 / \mathrm{mm}^{3}$, an albumin level of $2.5 \mathrm{~g} / \mathrm{dL}$, and a C-reactive protein level of $6.46 \mathrm{mg} / \mathrm{dL}$. Contrastenhanced whole-body computed tomography (CT) revealed liver and prostate abscesses (Fig. 1b and c), and contrast-enhanced head magnetic resonance imaging (MRI) revealed brain abscesses (Fig. 1d). However, a urine culture and two sets of blood cultures yielded negative results. K. pneumoniae was cultured from both the liver abscess specimen and the aqueous humor. Isolate identification was confirmed using the MicroScan WalkAway-96 SI system (Beckman Coulter Inc., Brea, CA, USA). The minimum inhibitory concentrations were also determined using the MicroScan WalkAway-96 SI. The K. pneumoniae was susceptible to all tested antimicrobials, with the exception of ampicillin. The agar plate colonies appeared hypermucoviscous and the string test yielded positive results (Fig. 1e). Therefore, we diagnosed the patient with hvKP invasive syndrome; brain, liver, and prostate abscesses; and bacterial endophthalmitis.

We performed enucleation of the right eyeball and percutaneous transhepatic drainage, and administered cefotaxime (6 g/day). Although the antibiotic treatment was continued for 9 days, it had little effect on the fever and inflammatory markers. Therefore, the antibiotic treatment was switched to levofloxacin (500 mg/day), and subsequently to meropenem (6 g/day). This reduced the fever and improved the inflammatory marker levels. However, the patient subsequently reported left lower quadrant abdominal pain. Contrast-enhanced abdominal CT revealed enlargement of an existing left internal iliac artery aneurysm (Fig. 1f), and we diagnosed the patient with infected hvKP aneurysm and impending rupture. Based on the patient's age and risk factors, we successfully performed coil embolization instead of surgery. He subsequently developed a rash over his body, which we attributed to the meropenem. Thus, the antibiotic regimen was switched to cefotaxime $(6 \mathrm{~g} /$ day) with levofloxacin $(500 \mathrm{mg} /$ day). After a 6 -week course of treatment, the patient's inflammatory marker levels returned to normal, and we observed reductions in the sizes of the brain, liver, and prostate abscesses. The antibiotic therapy is ongoing, and there has been no symptom recurrence.

\section{Case 2}

A 69-year-old man was referred to our hospital with a 10-day history of fever, pollakiuria, and pain on urination. He also exhibited vision loss in his left eye and a wet cough. The patient had a history of diabetes mellitus (DM) and hypertension, although there was no history of alcoholism. He had no recent travel history, including within Southeast Asia, and no history of contact with animals. A physical examination revealed that his blood pressure was $149 / 87 \mathrm{mmHg}$, his pulse was 122 beats per minute, his body temperature was $38.7{ }^{\circ} \mathrm{C}$, and his oxygen saturation was $93 \%$ in room air. We detected no abnormal findings during auscultation, and there was no costovertebral angle tenderness, although we detected corneal opacity in his left eye (Fig. 2a). Blood tests revealed a white blood cell count of $23,600 / \mathrm{mm}^{3}$, an albumin level of $2.6 \mathrm{~g} / \mathrm{dL}$, a C-reactive protein level of 26.22 $\mathrm{mg} / \mathrm{dL}$, and a hemoglobin A1c level of $8.1 \%$. Contrastenhanced whole-body $\mathrm{CT}$ revealed lung and prostate 

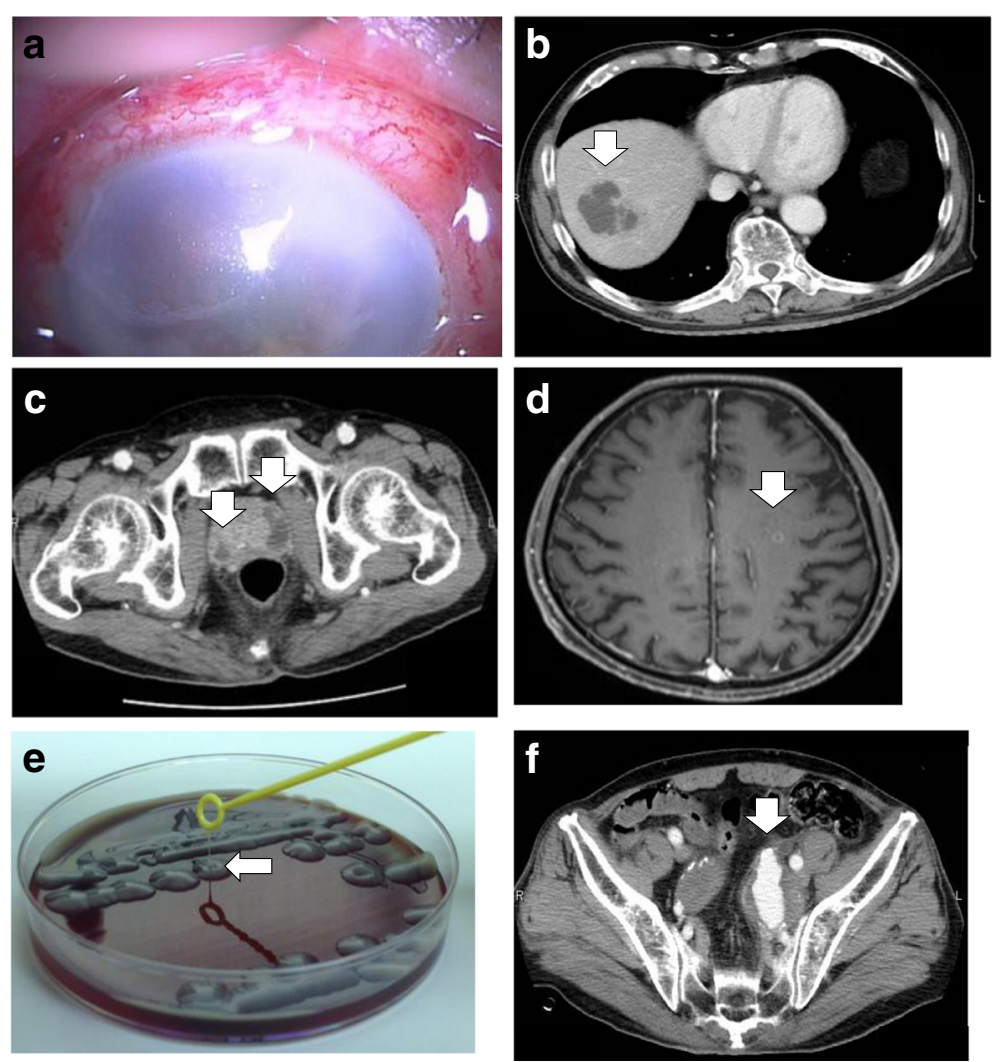

Fig. 1 Findings from the first case. a Findings of remarkable edema and opacity in the cornea. $\mathbf{b}$ Contrast-enhanced whole-body computed tomography (CT) reveals a liver abscess (white arrow). c Contrast-enhanced whole-body $\subset T$ reveals prostate abscesses (white arrows). $\mathbf{d}$ Contrast-enhanced head magnetic resonance imaging reveals a brain abscess (white arrow). e The first case provided colonies on the agar plate that stretched for $>5$ mm using the inoculation loop (white arrows), which indicates a positive string test result. $\mathbf{f}$ Contrast-enhanced whole-body CT reveals the enlargement of an existing left internal iliac artery aneurysm (white arrow)

abscesses (Fig. 2b and c), but no liver abscesses. Contrastenhanced head MRI revealed a brain abscess (Fig. 2d). $K$. pneumoniae was cultured from the sputum, urine, prostate abscess specimen, and aqueous humor. Isolate identification was confirmed using the MicroScan WalkAway-96 SI system (Beckman Coulter Inc., Brea, CA, USA). The minimum inhibitory concentrations were also determined using the MicroScan WalkAway-96 SI. The $K$. pneumoniae was susceptible to all tested antimicrobials, with the exception of ampicillin. The agar plate colonies appeared hypermucoviscous and the string test yielded positive results (Fig. 2e). Therefore, we diagnosed the patient with hvKP invasive syndrome; brain, lung, and prostate abscesses; and bacterial endophthalmitis.

We performed enucleation of the left eyeball and percutaneous drainage of the prostate abscess. We initially administered tazobactam/piperacillin (18 g/day), which was switched to intravenous levofloxacin (500 mg/day) based on drug migration to the prostate. The antibiotic regimen was continued for 7 days, although the patient's fever and inflammatory markers exhibited little improvement. Thus, we changed the antibiotic treatment to meropenem (6 g/day), which improved the fever and inflammatory markers, and we subsequently switched the patient to oral levofloxacin (500 $\mathrm{mg} /$ day) for maintenance therapy. After a 5-week course of treatment, we did not detect any markers of inflammation, and the brain, lung, and prostate abscesses had decreased in size. Therefore, the antibiotic treatment was discontinued and there has been no symptom recurrence.

\section{Genetic analysis}

Polymerase chain reaction (PCR) assays of the isolates from both cases were performed as previously described [9], and revealed the presence of $m a g A$ and $r m p A$ (Fig. 3). Multilocus sequence typing (MLST) was performed according to the methods of Diancourt et al. [10]. The MLST revealed sequence type 23 in both cases.

\section{Discussion}

K. pneumoniae is a common human pathogen that causes pneumonia and urinary tract infections (UTIs). In recent years, an increased incidence of hvKP syndrome has been reported in Taiwan [11], and this 

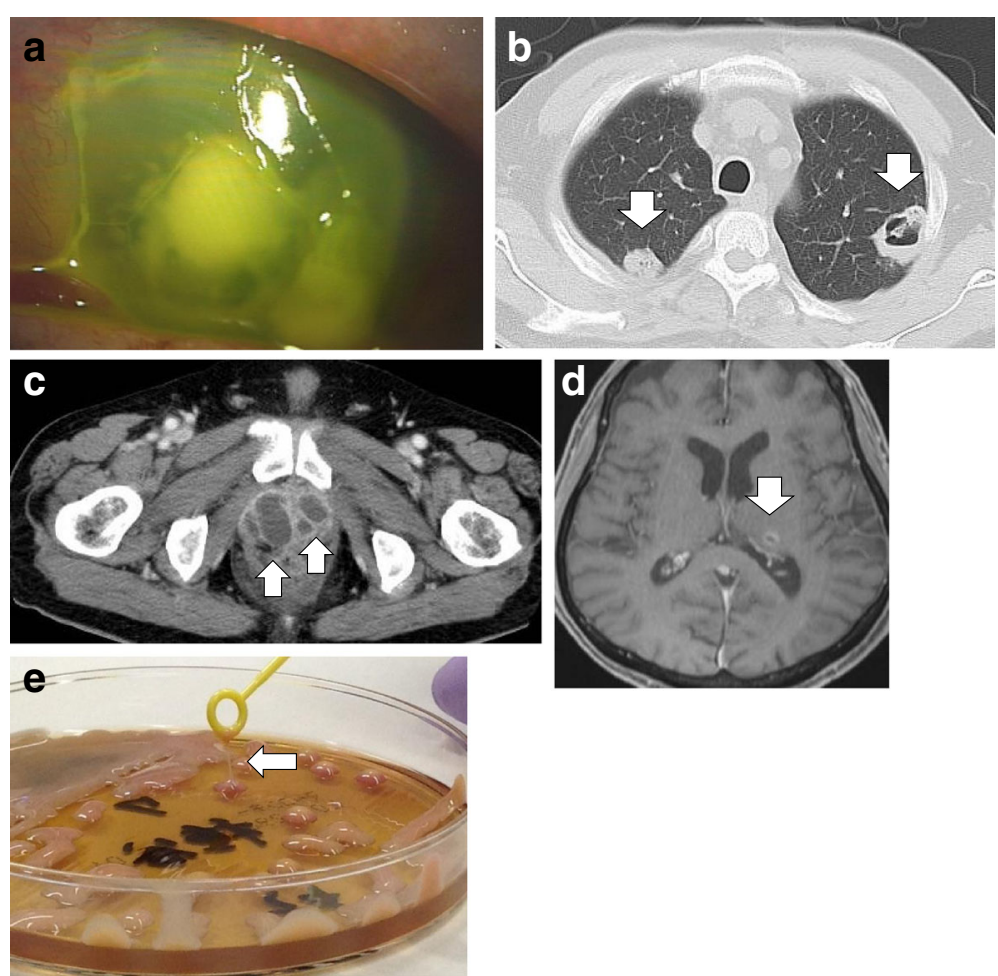

Fig. 2 Findings from the second case. a Findings of remarkable edema and opacity in the cornea. b Contrast-enhanced whole-body computed tomography (CT) reveals lung abscesses (white arrows). c Contrast-enhanced whole-body $C T$ reveals prostate abscesses (white arrows). d Contrastenhanced head magnetic resonance imaging reveals a brain abscess (white arrow). e The second case provided colonies on the agar plate that stretched for $>5 \mathrm{~mm}$ using the inoculation loop (white arrows), which indicates a positive string test result

syndrome is associated with bacteremia, primary liver abscess, and metastatic infections [6]. Extrahepatic complications have also been observed because of bacteremic dissemination, such as endophthalmitis, meningitis, and other diseases. However, to the best of our knowledge, there are few reports of infected aneurysms that were caused by hvKP $[12,13]$. In Case 1 from the present report, the patient reported new-onset left lower quadrant pain, and contrast-enhanced abdominal CT revealed enlargement of an existing left internal iliac artery aneurysm. Although we did not have positive culture results from the aneurysm tissue, the diagnosis of hvKP infection was made based on our clinical and diagnostic findings (newonset left lower quadrant pain and enlargement of the existing left internal iliac artery aneurysm despite appropriate antibiotic treatment). A previous report has indicated that DM and an age of $<65$ years were independent predictors of metastatic ocular or central nervous system complications of pyogenic liver abscesses [14]. Another report has identified a history of alcoholism and DM as significant risk factors for the development of metastatic complications from pyogenic liver abscesses [15]. However, the patient in Case 1 had no known risk factors, as he was 81 years old and had no history of DM or alcoholism. In addition, a previous report has indicated that glycemic control in patients with DM played an important role in the clinical characteristics of KP liver abscesses, especially in metastatic complications from KP liver abscess [16]. The patient in Case 2 took vildagliptin (100 mg/day) and his hemoglobin A1c was 8.1\%; therefore glycemic control was relatively poor. It is suggested that the poor glycemic control played an essential role in dissenminated KP infection.

The mechanisms for classic K. pneumoniae entry into extraintestinal sites include ascension from the perineum into the bladder, disruption of the bowel enabling entry of gastrointestinal tract colonizers into the peritoneal cavity, and aspiration of oropharyngeal colonizers into the respiratory tract [17]. However, the mechanism for hvKP remains unclear, and it is currently speculated that leakage of $K$. pneumoniae from a patient's intestinal mucosa, and bacterial translocation into the liver via the portal circulation, results in liver abscesses and bacteremic dissemination [6]. In Case 2, the patient presented with fever, pollakiuria, and pain on urination, which was indicative of a UTI. Furthermore, contrast-enhanced whole-body CT revealed prostate abscesses, but no liver abscesses. These findings suggested a primary prostate 


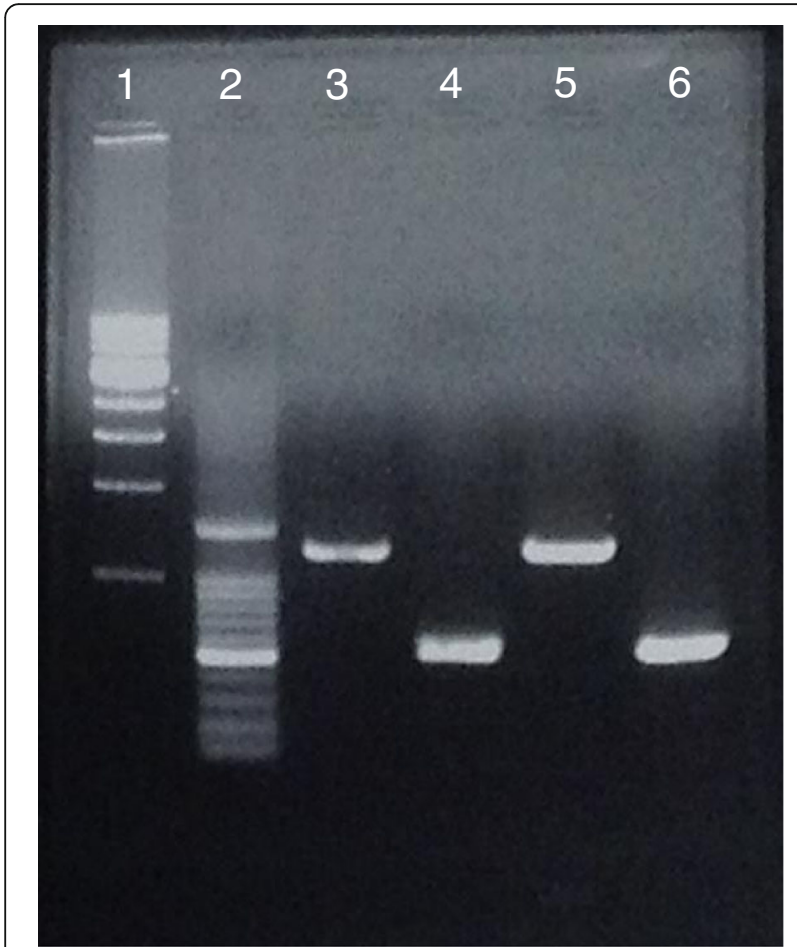

Fig. 3 Agarose gel electrophoresis. Lane 1: 1-10 kbp DNA ladder; Lane 2: 100-1,500 bp DNA ladder; Lanes 3 and 4: the PCR product reveals $m a g A$ and $r m p A$ in the first patient; Lanes 5 and 6 : the PCR product reveals magA and $r m p A$ in the second patient

lesion, although the ascending route of infection via the prostate is an extremely rare cause of hvKP bacteremia. Moreover, this patient presented with disseminated lesions secondary to the prostate infection, which is extremely rare. Unlike liver abscesses, the $\mathrm{K} 1$ serotype of $\mathrm{K}$. pneumoniae is considered closely related to hematogenous metastasis, and is rarely detected in the urinary tract [18]. In addition, clinicians may reach a rapid diagnosis of a UTI based on its typical symptoms, such as micturition pain, constant urge to urinate, and back pain. Therefore, we speculate that there are few reports of UTIs that were caused by hvKP, as antibiotic treatment would typically be started before the development of disseminated abscesses.

MLST has been used as a nucleotide sequence-based method for characterizing microorganisms [19]. This approach is particularly useful for the typing of microbial pathogens, in order to identify clones with noticeably different virulence characteristics [20]. Invasive syndrome caused by highly virulent strains with sequence type 23 has become increasingly common in Southeast Asia [21], and the MLST revealed sequence type 23 in both of the present cases.

The hypermucoviscous phenotype can easily be confirmed using the string test [6]. In this context, a positive result is defined as bacterial colonies on an agar plate stretching for $>5 \mathrm{~mm}$ using the inoculation. Thus, both of the present cases exhibited positive results for the hypermucoviscous phenotype, based on the string test results. Furthermore, the $m a g A$ and $\operatorname{rmp} A$ genes are related to the expression of the hypermucoviscous phenotype [7]. In this context, the magA gene encodes an outer membrane protein that is essential for the formation of a protective exopolysaccharide web, which is related to the bacterial virulence and mucoviscosity of the K1 serotype [22]. The enzyme encoded by magA gene functions as a polymerase involved in capsule synthesis, and this function is restricted to the capsular gene cluster of $\mathrm{K} 1$ serotype only [6]. In contrast, the $r m p A$ gene is a plasmid-borne regulator of extracellular polysaccharide synthesis [23]. Moreover, the $r m p A$ gene is strongly associated with abscess formation [7].

\section{Conclusions}

In conclusion, we report two rare cases of hvKP invasive syndrome that involved strains that were positive for $m a g A$ and $\operatorname{rmp} A$. Therefore, we suggest that a diagnosis of hvKP invasive syndrome should be considered in all patients who present with $K$. pneumoniae infection and multiple organ abscesses.

\section{Abbreviations}

CT: computed tomography; DM: diabetes mellitus; hvKP: hypermucoviscous K. pneumoniae; K. pneumoniae: Klebsiella pneumoniae; magA: mucoviscosityassociated gene A; MLST: multilocus sequence typing; MRI: magnetic resonance imaging; $P C R$ : polymerase chain reaction; $r m p A$ : the regulator of mucoid phenotype A; UTI: urinary tract infection

\section{Acknowledgements}

This research was supported by the Research Program on Emerging and Reemerging Infectious Diseases from the Japanese Agency for Medical Research and Development.

\section{Funding \\ None.}

\section{Availability of data and materials}

Seven housekeeping genes (gapA, $m d h, g p i, \operatorname{rop} B$, inf, phoE, and tonB) were amplified, sequenced, and compared with available sequences from the MLST website (http://bigsdb.web.pasteur.fr/klebsiella/klebsiella.html).

\section{Authors' contributions}

All authors contributed equally to: 1) the interpretation of the clinical data, 2) the drafting of the manuscript and revising it critically, and 3) the final approval of the submitted manuscript.

\section{Competing interests}

The authors declare that they have no competing interests.

\section{Ethics approval and consent to participate}

Written informed consent was obtained from both patients for the publication of this case report and the accompanying images.

\section{Author details}

${ }^{1}$ Department of Infection Control Science, Osaka City University, Graduate School of Medicine, 1-4-3, Asahi-machi, Abeno-ku, Osaka 545-8585, Japan. ${ }^{2}$ Department of Medical Education and General Practice, Osaka City University, Graduate School of Medicine, 1-4-3, Asahi-machi, Abeno-ku, Osaka 545-8585, Japan. ${ }^{3}$ Department of Bacteriology, Osaka City University, Graduate School of Medicine, 1-4-3, Asahi-machi, Abeno-ku, Osaka 545-8585, Japan. 
Received: 22 January 2016 Accepted: 7 November 2016

Published online: 16 November 2016

\section{References}

1. Podschun R, Ullmann U. Klebsiella spp. as nosocomial pathogens: epidemiology, taxonomy, typing methods, and pathogenicity factors. Clin Microbiol Rev. 1998; 11(4):589-603.

2. Chang FY, Chou MY, Fan RL, Shaio MF. A clinical study of Klebsiella liver abscess. Taiwan Yi Xue Hui Za Zhi. 1988:87(3):282-7.

3. Liu YC, Cheng DL, Lin CL. Klebsiella pneumoniae liver abscess associated with septic endophthalmitis. Arch Intern Med. 1986;146(10):1913-6.

4. Nakamoto K, Koide T, Nagatomo T, Tamura M, Higaki M, Takata S, et al. Severe primary liver abscess and septic pulmonary embolism due to Klebsiella pneumoniae with hypermucoviscosity phenotype. Kansenshogaku Zasshi. 2011; 85(4):366-9 [In Japanese].

5. Maruno T, Ooiwa Y, Takahashi K, Kodama Y, Takakura S, Ichiyama S, et al. A liver abscess deprived a healthy adult of eyesight: endogenous endophthalmitis associated with a pyogenic liver abscess caused by serotype K1 Klebsiella pneumonia. Intern Med. 2013;52(8):919-22.

6. Siu LK, Yeh KM, Lin JC, Fung CP, Chang FY. Klebsiella pneumoniae liver abscess: a new invasive syndrome. Lancet Infect Dis. 2012;12(11):881-7.

7. Yu WL, Ko WC, Cheng KC, Lee HC, Ke DS, Lee CC, et al. Association between rmpA and magA genes and clinical syndromes caused by Klebsiella pneumoniae in Taiwan. Clin Infect Dis. 2006:42(10):1351-8.

8. Kawai T. Hypermucoviscosity: an extremely sticky phenotype of Klebsiella pneumoniae associated with emerging destructive tissue abscess syndrome. Clin Infect Dis. 2006;42(10):1359-61.

9. El Fertas-Aissani R, Messai Y, Alouache S, Bakour R. Virulence profiles and antibiotic susceptibility patterns of Klebsiella pneumoniae strains isolated from different clinical specimens. Pathol Biol. 2013;61(5):209-16.

10. Diancourt L, Passet V, Verhoef J, Grimont PA, Brisse S. Multilocus sequence typing of Klebsiella pneumoniae nosocomial isolates. J Clin Microbiol. 2005; 43(8):4178-82.

11. Fang CT, Lai SY, Yi WC, Hsueh PR, Liu KL, Chang SC. Klebsiella pneumoniae genotype K1: an emerging pathogen that causes septic ocular or central nervous system complications from pyogenic liver abscess. Clin Infect Dis. 2007:45(3):284-93.

12. Chuang YC, Lee MF, Yu WL. Mycotic aneurysm caused by hypermucoviscous Klebsiella pneumoniae serotype K54 with sequence type 29: an emerging threat. Infection. 2013;41(5):1041-4.

13. Chen YJ, Chen SY, Wang JT, Hsueh PR. Mycotic aneurysm caused by gas-forming serotype K5 Klebsiella pneumoniae. Int J Infect Dis. 2009; 13(2):e47-8.

14. Lin YT, Liu CJ, Chen TJ, Fung CP. Long-term mortality of patients with septic ocular or central nervous system complications from pyogenic liver abscess: a population-based study. PLoS One. 2012;7(3), e33978.

15. Chen SC, Lee YT, Lai KC, Cheng KS, Jeng LB, Wu WY, et al. Risk factors for developing metastatic infection from pyogenic liver abscesses. Swiss Med Wkly. 2006;136(7-8):119-26.

16. Lin YT, Wang FD, Wu PF, Fung CP. Klebsiella pneumoniae liver abscess in diabetic patients: association of glycemic control with the clinical characteristics. BMC Infect Dis. 2013;13:56.

17. Shon AS, Bajwa RP, Russo TA. Hypervirulent (hypermucoviscous) Klebsiella pneumoniae: a new and dangerous breed. Virulence. 2013:4(2):107-18.

18. Tsay RW, Siu LK, Fung CP, Chang FY. Characteristics of bacteremia between community-acquired and nosocomial Klebsiella pneumoniae infection: risk factor for mortality and the impact of capsular serotypes as a herald for community-acquired infection. Arch Intern Med. 2002; 162(9):1021-7

19. Maiden MC, Bygraves JA, Feil E, Morelli G, Russell JE, Urwin R, et al. Multilocus sequence typing: a portable approach to the identification of clones within populations of pathogenic microorganisms. Proc Natl Acad Sci USA. 1998;95(6):3140-5

20. Zhao J, Chen J, Zhao M, Qiu X, Chen X, Zhang W, et al. Multilocus sequence types and virulence determinants of hypermucoviscosity-positive Klebsiella pneumoniae isolated from community-acquired infection cases in Harbin, North China. Jpn J Infect Dis. 2016;69(5):357-60.

21. Gundestrup S, Struve C, Stahlhut SG, Hansen DS. First Case of Liver Abscess in Scandinavia Due to the International Hypervirulent Klebsiella Pneumoniae Clone ST23. Open Microbiol J. 2014;8:22-4.
22. Fang $C T$, Chuang YP, Shun $C T$, Chang SC, Wang JT. A novel virulence gene in Klebsiella pneumoniae strains causing primary liver abscess and septic metastatic complications. J Exp Med. 2004;199(5):697-705.

23. Compain F, Babosan A, Brisse S, Genel N, Audo J, Ailloud F, et al. Multiplex $P C R$ for detection of seven virulence factors and K1/K2 capsular serotypes of Klebsiella pneumoniae. J Clin Microbiol. 2014;52(12):4377-80.

\section{Submit your next manuscript to BioMed Central and we will help you at every step:}

- We accept pre-submission inquiries

- Our selector tool helps you to find the most relevant journal

- We provide round the clock customer support

- Convenient online submission

- Thorough peer review

- Inclusion in PubMed and all major indexing services

- Maximum visibility for your research

Submit your manuscript at www.biomedcentral.com/submit
Biomed Central 